Reprod. Nutr. Dévelop., 1980, 20 (4 B), 1369-1377.

\title{
Toxicité comparée de différentes céréales pour les sujets intolérants au gluten
}

\author{
par L. CHARBONNIER, J. JOS *, J. F. MOUGENOT *, J. MOSSÉ \\ avec la collaboration technique de Claude DEMARTEAU, \\ M. SALLANTIN ef J. C. HUET \\ Laboratoire d'Etude des Protéines, \\ Département de Physiologie ef Biochimie végétales, I.N.R.A., \\ route de Saint-Cyr, 78000 Versailles \\ * Unité de Recherches de Génétique médicale \\ U 12 (INSERM) Hôpital des Enfants Malades, 75730 Paris
}

Summary. Comparative toxicity of different cereals for gluten-intolerant individuals.

Coeliac disease is caused by prolamines, the storage proteins of some cereals, located in the endosperm. Cereals do not all have the same toxicity.

The four wheat prolamine groups $(\alpha, \beta, \gamma$ and $\omega$ gliadins), visible in electrophoresis at acid $\mathrm{pH}$, have been isolated and their toxicities compared by observing the morphological changes in intestinal biopsies cultured in vitro when peptic-tryptic digests of the studied proteins were added to the culture medium. The toxicity was found to be mainly located in the $\alpha$ and $\beta$-gliadins and in peptides of 5 to 10000 molecular weight.

Peptides, resulting from peptic-tryptic hydrolysis, varied in length as a direct function of their proline content. In fact, peptide bond splitting by pepsin and trypsin is known to be blocked by proline. Thus, proline content determines peptide length and toxicity.

Wheat, rye and barley toxicities were compared on the basis of a correlation between toxicity and the $\alpha$ - and $\beta$-gliadin-like prolamine contents of these cereals.

Electrophoretic estimation of $\alpha$ - and $\beta$-gliadin-like prolamine content gave the following prediction of relative toxicity (in decreasing order) : wheat, friticale, rye, barley and oats.

\section{Introduction.}

La maladie coliaque (ou intolérance au gluten) est due à l'ingestion de certaines céréales. Elle se traduit par des phénomènes de malabsorption de presque tous les éléments nutritifs. Cette maladie concerne environ une personne sur 2000 , tant en Europe de l'Ouest qu'aux Etats-Unis (McCrae, 1970 ; Kasarda, 1978), mais peut affecter une personne sur 300 dans l'Ouest de l'Irlande (Mylotte ef al., 1973) et même une sur 250 en Afrique du Nord.

Les céréales connues pour leur toxicité sont, par ordre de toxicité décroissante, le Blé, le Seigle et l'Orge. La toxicité de l'Avoine est très controversée (Dissanayake, Truelove et Whitehead, 1974 ; Baker ef Read, 1976). 
Dans le présent travail, des explications biochimiques ont été recherchées pour tenter d'expliquer les différences de toxicité entre céréales.

\section{Matériel et méthodes.}

L'extraction de la gliadine à partir de Blé Cappelle a été effectuée suivant le protocole décrit par Charbonnier (1970). Une farine préalablement délipidée par agitation dans du n-butanol saturé d'eau est agitée dans de l'éthanol à 55 p. $100(p / p)$. Le surnageant obtenu après centrifugation est dilué 3 fois avec une solution à $1,5 p .100$ de $\mathrm{NaCl}$. Le précipité obtenu après un séjour de $48 \mathrm{~h}$ de la suspension à $4^{\circ} \mathrm{C}$ est ensuite séché par l'éthanol absolu, puis sous vide en présence de $\mathrm{H}_{2} \mathrm{SO}_{4}$. C'est la gliadine totale.

La $\gamma$-gliadine a été isolée en fractionnant la gliadine totale par chromatographie sur Sulfopropyl Séphadex G50 (Charbonnier et Mossé, 1980).

Les électrophorèses sur gel d'amidon ont été effectuées en utilisant un tampon Urée $3 \mathrm{M}$, lactate d'aluminiu m $0,0015 \mathrm{M}, \mathrm{pH} 3,2$ suivant le protocole décrit par Landry ef al. (1965).

Les hydrolyses pepsiques-trypsiques (PT) ont porfé sur $500 \mathrm{mg}$ de $\gamma$-gliadine suivant le protocole de Jos et al. (1975). Les enzymes ont été fournies par Sigma.

Les masses molaires des peptides PT ont été déterninées par filtration sur gel (Andrews, 1964) en utilisant une colonne $(2,6 \times 55 \mathrm{~cm})$ de Séphadex G50 équilibré avec un tampon laurylsulfate de sodium $0,05 \mathrm{M}$, parachloromercuribenzoate $10^{-4} \mathrm{M}$, Tris- $\mathrm{HCl}$ pH 8 (Pagé et Godin, 1969). La colonne a été étalonnée avec les protéines ou peptides suivants : inhibiteur trypsique du haricot de lima (Sigma, $M=9000$ ), insuline (Sigma, $M=5750$ ) et glucagon (Sigma, $M=3500$ ).

On a posé sur la colonne des échantillons de $2 \mathrm{ml}$ à $0,15 \mathrm{p}$. 100 de protéines étalons ou 0,25 à 0,4 p. 100 de peptides PT.

La colonne a été éluée à un débit de $12 \mathrm{ml} / \mathrm{h}$ et on a recueilli des fractions de $4 \mathrm{ml}$ dont les absorptions ont été mesurées à $280 \mathrm{~nm}$.

Les analyses d'acides aminés ont été effectuées au moyen d'analyseurs automafiques Phoenix K 8000 . Tous les échantillons $(5-7 \mathrm{mg})$ ont été hydrolysés à $110^{\circ} \mathrm{C}$ dans des tubes scellés sous vide. Les acides aminés acides, neutres et basiques ont été déterminés après des hydrolyses de 24 et $48 \mathrm{~h}$. Les acides aminés soufrés ont été déterminés après oxydation performique.

Les teneurs en azole des fractions peptidiques ont été estimées d'après les résullats des analyses d'acides aminés en supposant que les rendements des chromatographies étaient de 100 p. 100.

Enfin, le protocole des tests de toxicité in vitro par culture organotypique a déjà été décrit par Jos et al. (1975). Ces tests consistent à apprécier la toxicité d'un hydrolysat pepsique-trypsique de protéine d'après la réaction d'une biopsie de muqueuse intestinale cultivée in vitro en présence de cet hydrolysat dans le milieu de culture. Les biopsies sont prélevées sur des malades non traités ou en rechute. La toxicité s'exprime sur ces muqueuses altérées par une inhibition de la réparation épithéliale, voire par une nécrose massive de l'épithélium (Jos ef Rey, 1975). 


\section{Résultats.}

Fractionnement de l'hydrolysat pepsique-trypsique par chromatographie sur Sépha$\operatorname{dex}$ G 50. - Le fractionnement de l'hydrolysat PT de $\gamma$-gliadine sur Séphadex G50 est représenté sur la figure 1 . On compte 8 fractions plus ou moins bien individualisées.

FIG. 1. - Fractionnement sur Sëphadex $G 50$ d'un hydrolysat pepsique-trypsique de Y-gliadine.

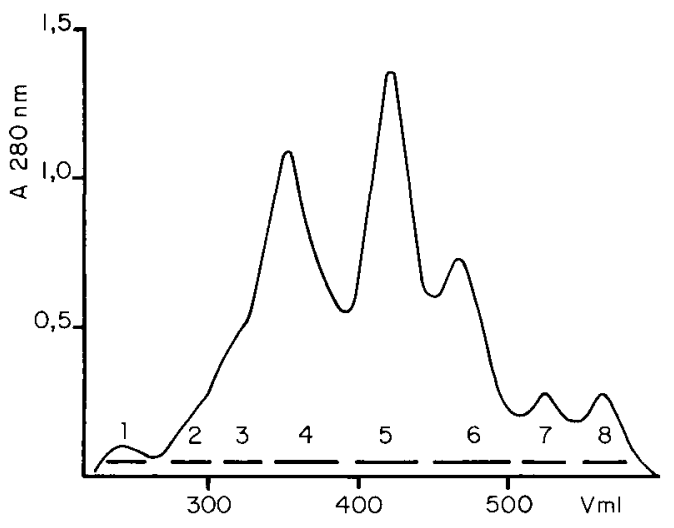

Caractérisation des peptides pepsiques-trypsiques. - Les compositions en acides aminés des fractions peptidiques, leurs masses molaires ef leurs teneurs en azote sont données dans le tableau 1, ainsi que la composition en acides aminés de la $\gamma$-glia-

\section{TABLEAU 1}

Compositions en acides aminés (nombre de résidus p. 100), masses molaires $(M)$ et teneurs en azote (N/MS p. 100) des peptides PT de $\gamma$-gliadine séparés sur Séphadex G50

\begin{tabular}{|c|c|c|c|c|c|c|c|}
\hline \multirow[b]{2}{*}{ A. Aminés } & \multirow[b]{2}{*}{$\gamma-G^{*}$} & \multicolumn{6}{|c|}{ Fractions G 50} \\
\hline & & 1 & 2 & 3 & 4 & 5 & 6 \\
\hline 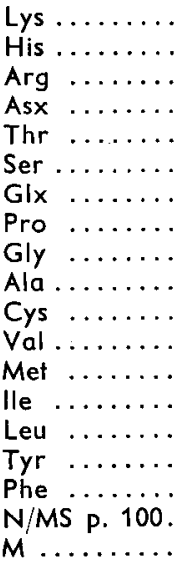 & $\begin{array}{r}5 \\
12 \\
12 \\
18 \\
21 \\
47 \\
403 \\
175 \\
27 \\
31 \\
24 \\
41 \\
11 \\
45 \\
70 \\
7 \\
51\end{array}$ & $\begin{array}{c}0 \\
7 \\
5 \\
2 \\
33 \\
12 \\
488 \\
310 \\
3 \\
7 \\
1 \\
9 \\
1 \\
22 \\
0 \\
100 \\
13,2\end{array}$ & $\begin{array}{c}1 \\
4 \\
6 \\
2 \\
34 \\
10 \\
486 \\
313 \\
2 \\
6 \\
5 \\
4 \\
1 \\
3 \\
18 \\
1 \\
104 \\
14,6 \\
11000\end{array}$ & $\begin{array}{c}6 \\
14 \\
2 \\
16 \\
30 \\
33 \\
419 \\
236 \\
10 \\
22 \\
28 \\
41 \\
7 \\
19 \\
54 \\
6 \\
57 \\
12,6 \\
7200\end{array}$ & $\begin{array}{c}10 \\
16 \\
8 \\
26 \\
22 \\
48 \\
370 \\
200 \\
12 \\
31 \\
49 \\
44 \\
15 \\
32 \\
63 \\
16 \\
38 \\
13,8 \\
5400\end{array}$ & $\begin{array}{c}6 \\
16 \\
19 \\
38 \\
12 \\
96 \\
248 \\
90 \\
74 \\
59 \\
14 \\
78 \\
13 \\
89 \\
115 \\
6 \\
27 \\
12,8 \\
4500\end{array}$ & $\begin{array}{c}5 \\
4 \\
100 \\
37 \\
26 \\
109 \\
232 \\
56 \\
37 \\
83 \\
6 \\
59 \\
15 \\
81 \\
89 \\
20 \\
41 \\
3,8 \\
2700\end{array}$ \\
\hline
\end{tabular}

* $\gamma$-Gliadine. 
dine. Les fractions 7 ef 8 mises en évidence par chromatographie sur Séphadex G50 (fig. 1) n'ont pas été caractérisées en raison de la trop faible quantité de matériel récupérée. La faible teneur en azote de la fraction 6 est probablement due à une proportion élevée de glucides.

Electrophorèses sur gel d'amidon. - Les diagrammes électrophorétiques des prolamines du Blé (gliadine), du Triticale (Triticaline), du Seigle (Sécaline), de l'Orge (Hordéine) et de l'Avoine (Avénine), sont représentés sur la figure 2.
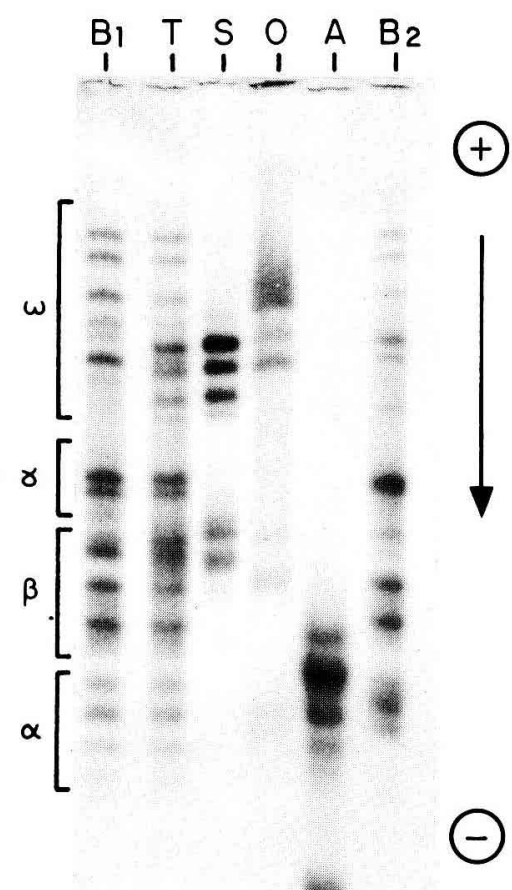

FIG. 2. - Electrophorèses sur gel d'amidon à $\mathrm{pH} \quad 3,2$ de gliadines $\left(B_{1}\right.$ et $\left.B_{2}\right)$, Trificaline $(T)$, Sécaline $(S)$ Hordéine $(O)$ et Avénine $(A)$.

On a utilisé la nomenclature proposée par Woychik, Boundy et Dimler (1961) pour désigner les 4 groupes de constituants de la gliadine mis en évidence par électrophorèse à $\mathrm{pH}$ acide.

Les groupes de constituants de même mobilité électrophorétique ont été désignés par les mêmes lettres $\alpha, \beta, \gamma$ et $\omega$, quelle que soit la prolamine à laquelle ils appartiennent, sauf dans le cas des avénines qui pouvaient être considérées comme un tout, en raison des très faibles différences de composition en acides aminés des 2 groupes de constituants mis en évidence par électrophorèse à pH acide (Kim, Charbonnier et Mossé, 1978).

Composition en acides aminés des gliadines $\alpha, \beta, \gamma$ et $\omega$. - Les résultats sont présentés dans le tableau 2. Les sommes des acides aminés basiques des 4 groupes de constituants sont bien en accord avec leurs mobilités relatives en électrophorèse à pH acide. 
Les teneurs en Asx et Glx permettent de calculer les rapports $N$ amidé/ $N$ aminé des 4 groupes de gliadines suivant que les résidus Asx et Glx sont amidés à 100 ou 90 p. 100 (Ewart, 1967) (cf. tabl. 3).

\section{TABLEAU 2}

Composition en acides aminés partielle des gliadines testées in vitro (Nombre de résidus p. 1000 résidus retrouvés)

\begin{tabular}{lccrr}
\hline \multicolumn{1}{l}{ Ac. aminés } & $\omega$ & $\gamma$ & & \\
& & & & $\alpha$ \\
\hline Lys $\ldots \ldots \ldots \ldots$ & 4 & 5,6 & 4 & 5 \\
His $\ldots \ldots \ldots \ldots$ & 9,5 & 14 & 15 & 24 \\
Arg $\ldots \ldots \ldots \ldots$ & 6,5 & 12,5 & 17 & 21 \\
Asx $\ldots \ldots \ldots \ldots$ & 5 & 19 & 25 & 28 \\
Glx $\ldots \ldots \ldots \ldots$ & 501 & 391 & 378 & 370 \\
Pro $\ldots \ldots \ldots \ldots$ & 229 & 181 & 165 & 151 \\
& & & & \\
\hline
\end{tabular}

\section{Discussion.}

Dicke (1950) ef Van de Kamer, Weijers ef Dicke (1953) ont montré que dans le cas du Blé, la toxicité était imputable aux gliadines, protéines spécifiques de l'albumen. Les gliadines appartiennent au groupe des prolamines qui doivent leur nom à leur richesse particulière en 2 acides aminés : la proline et la glutamine (Mossé, 1966).

Ayant observé que la désamidation de la gliadine supprimait sa toxicité, Van de Kamer et Weijers (1955) ont proposé de classer les protéines par ordre de toxicité en fonction de leur teneur en azote amidé lié. lls constatent que pour des valeurs du rapport $\mathrm{N}$ amidé/ $\mathrm{N}$ aminé supérieures à 11 , les protéines sont d'autant plus toxiques que le rapport est élevé ; la valeur la plus élevée étant celle trouvée pour la gliadine. Or, si l'on calcule les valeurs de ce rapport pour les groupes de gliadines $\alpha, \beta, \gamma$ et $\omega$ mis en évidence par électrophorèse de gliadine totale à $\mathrm{pH}$ acide, c'est pour le groupe des $\omega$ que l'on trouve la valeur la plus élevée (tabl. 3) alors que d'après les résultats des tests in vitro, visant à comparer les toxicités des 4 groupes de gliadines, ce sont

TABLEAU 3

Rapport $N$ amide/ $N$ amine (p. 100) des différentes gliadines en fonction des taux d'amidation des résidus Asx et $G / x$

\begin{tabular}{|c|c|c|c|c|}
\hline \multirow{2}{*}{ Amidation } & \multicolumn{4}{|c|}{ Gliadines } \\
\hline & $\omega$ & $\gamma$ & $\beta$ & $\alpha$ \\
\hline $\begin{aligned} 100 & \text { p. } 100 \ldots \ldots \\
90 & \text { p. } 100 \ldots \ldots\end{aligned}$ & $\begin{array}{l}48 \\
44\end{array}$ & $\begin{array}{l}38 \\
34\end{array}$ & $\begin{array}{l}37 \\
33\end{array}$ & $\begin{array}{l}36 \\
32\end{array}$ \\
\hline
\end{tabular}


précisément les $\omega$-gliadines qui accusent la toxicité la plus faible (tabl. 4). Le degré d'amidation d'une protéine est donc insuffisant pour mesurer sa toxicité.

TABLEAU 4

Toxicités in vitro d'hydrolysats pepsiques trypsiques *

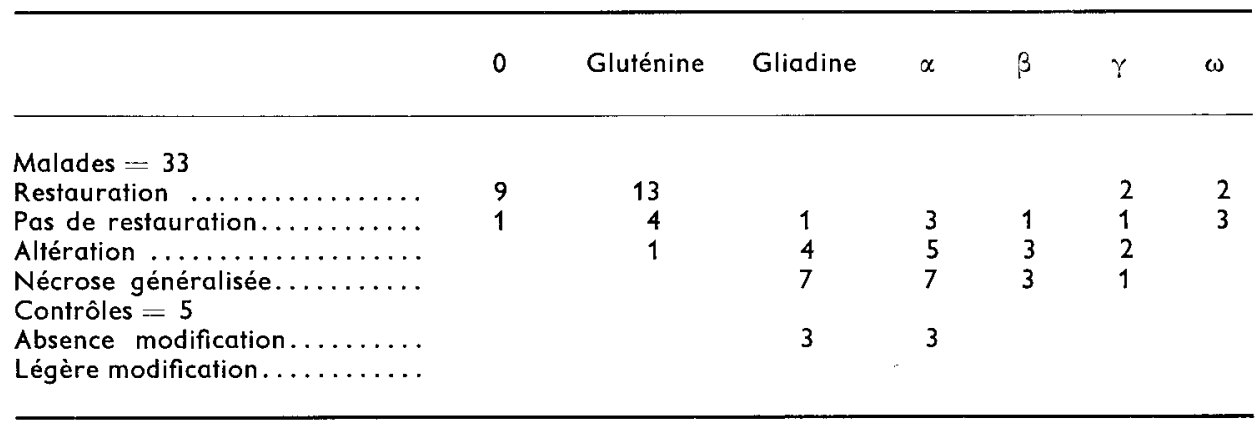

* Jos ef al. (1978).

Nous proposons d'expliquer la toxicité des prolamines non seulement par leur teneur en acides aminés porteurs de fonctions amides (Gln et Asn), mais également par leur teneur en proline.

Des études récentes (Dissanayake et al., 1973 ; Jos et Rey, 1975) ont en effet montré qu'il y avait une relation entre toxicité et longueur des peptides résultant d'une hydrolyse de gliadine totale par la pepsine ef la trypsine. Seuls les peptides ayant des masses molaires comprises entre 5 et 10000 daltons sont toxiques.

On sait d'autre part que la pepsine ef la trypsine ne coupent pas les liaisons peptidiques comprenant une proline (Smyth, 1967). Les différences de teneurs en proline sont donc toules indiquées pour expliquer les différences de taille entre peptides résultant de l'hydrolyse d'une protéine par la pepsine ef la trypsine. Nous l'avons vérifié dans le cas de la $\gamma$-gliadine, en comparant les compositions en acides aminés ef les masses molaires des peptides séparés par chromatographie sur Séphadex G50 d'un hydrolysat de $\gamma$-gliadine (fig. 1). On constate que les peptides les plus longs sont ceux dont les teneurs en proline sont les plus élevées (tabl. 1).

Des teneurs en proline, ainsi que des poids moléculaires des chaînes polypeptidiques de gliadines dépendent donc les rendements de leur hydrolyse pepsiquetrypsique en peptides d'une taille définie.

Les $\omega$-gliadines, qui allient des teneurs en proline de l'ordre de 23 p. 100 à des poids moléculaires doubles de ceux des gliadines $\alpha$ ef $\beta$ dont les teneurs en proline sont de l'ordre de 15 à 16 p. 100 (tabl. 2) (Charbonnier, 1974 ; Laforgue-Tercé, 1979) donnent ainsi par hydrolyse pepsique-trypsique, une proportion élevée de peptides de haut poids moléculaires non toxiques (Jos, données non publiées).

La toxicité d'une céréale serait donc liée à la présence dans son albumen, non pas de prolamines à taux d'amidation élevé (type $\omega$ ), mais de prolamines ayant des caractéristiques proches de celles des gliadines $\alpha$ et $\beta: 15$ à 16 p. 100 de proline et des poids moléculaires de 30 à 35000 (Laforgue-Tercé, 1979). 
De telles prolamines ont été mises en évidence dans le Seigle (Preston et Woodbury, 1975 ; Charbonnier, données non publiées). Ce sont les sécalines de mêmes mobilités électrophorétiques que les $\beta$-gliadines les plus lentes (fig. 2 ). On en trouve également dans l'Orge. Ce sont les Hordéines de même mobilité que les $\alpha$-gliadines (Laurière, Charbonnier et Mossé, 1976 ; Shewry ef al., 1979 ; Laurière, données non publiées) (fig. 2). $\alpha$ - et $\beta$-gliadines ainsi que $\beta$-sécalines sont également présentes dans le Triticale, amphiploïde obtenu à partir du Blé et du Seigle. Le diagramme électrophorétique de la Trificaline résulte en effet de l'addition des diagrammes électrophorétiques des prolamines des deux parents. L'Avoine, par contre, est dépourvue de tels constituants. Les teneurs en proline des avénines sont de l'ordre de 10 p. 100 et leurs masses molaires voisines de 22000 (Kim, Charbonnier et Mossé, 1978).

Enfin, le niveau de toxicité d'une céréale ne dépend pas seulement de la nature de ses prolamines mais également de la teneur du grain en prolamines toxiques. Les données quantitatives concernant les prolamines des différentes céréales sont malheureusement encore insuffisantes, en dehors des cas du Blé et du Seigle, pour que l'on puisse calculer les teneurs en prolamines toxiques ou supposées comme telles (tabl. 5).

\section{TABLEAU 5}

Teneurs en prolamines ef foxicité

du Blé, du Triticale, du Seigle, de l'Orge et de l'Avoine

\begin{tabular}{|c|c|c|c|c|c|}
\hline & Blé & Triticale & Seigle & Orge & Avoine \\
\hline Protéines totales & 12 & 9,8 & 9,9 & 10 & 10 \\
\hline Grain p. 100 & (1) & (1) & (1) & (3) & (4) \\
\hline Prolamines & 41 & 24 & 19 & 15 & 11 \\
\hline Protéines totales $P .100 . . . \ldots$ & & & & & (5) \\
\hline$\frac{\text { Prolamines }}{\text { Grain }}$ p. $100 \ldots \ldots \ldots \ldots$ & 4,9 & 2,35 & 1,9 & 1,5 & 1,1 \\
\hline$\frac{\text { Prol. toxiques }}{\text { Prolamines }}$ p. $100 \ldots \ldots \ldots \ldots$ & $\begin{array}{l}55 \\
(2)\end{array}$ & - & 7 & - & ? \\
\hline$\frac{\text { Prol. toxiques }}{\text { Grain }}$ p. 100. & 2,7 & - & 0,1 & - & $?$ \\
\hline Toxicités prévues........... & $t+t+$ & +++ & $+t$ & + & 0 \\
\hline Toxicités connues........... & $+t^{-t}+$ & $?$ & $+t^{-}$ & + & $?$ \\
\hline
\end{tabular}

(1) Chen et Bushuk (1970).

(2) Charbonnier et Mossé (1980).

(3 et 4) Bourdet $(1956 a, 1956 b)$.

(5) $\operatorname{Kim}(1978)$.

On peut toutefois estimer les proportions des groupes de constituants des différentes prolamines d'après leurs diagrammes électrophorétiques. La proportion de prolamines dans le Triticale (tabl. 5) situe sa toxicité entre celles du Blé et du Seigle, compte-tenu des proportions des constituants ( $\alpha$ et $\beta$ ) dans la triticaline. D'après ses 
caractéristiques, l'Orge serait moins toxique que le Seigle. En effet, au vu des diagrammes électrophorétiques, la proportion d'Hordéines $\alpha$ dans l'hordéine totale est inférieure à celle des $\beta$-sécalines dans la prolamine du Seigle et d'autre part, la teneur en prolamines de l'Orge est inférieure à celle du Seigle. Nous avons vu enfin que, compte tenu de leurs propriétés physicochimiques, la toxicité des avénines était fort peu probable. Elle est d'ailleurs très contestée (Dissanayake, Truelove ef Whitehead, 1974).

\section{Conclusions.}

Il semble par conséquent possible de classer les céréales par ordre de toxicité d'après les caractéristiques physicochimiques et les quantités de certaines des prolamines présentes dans leur albumen. L'ordre des toxicités prévues est en effet le même que celui des toxicités connues. La teneur en proline des chaînes polypeptidiques paraît déterminer au moins partiellement le caractère de toxicité.
Journées Ingestion-Digestion-Absorption de l'Association française de Nutrition,
Paris, 15-16 novembre 1979.

Remerciements. - Ce travail a bénéficié de l'aide de I'INSERM (contrat ATP 74.79.106).

\section{Références}

ANDREWS P., 1964. Estimation of the molecular Weights of proteins by Sephadex Gel-Filtration. Biochem. J., 91, 222-233.

BAKER P. G., READ A. E., 1976. Oats and barley toxicity in coeliac patients. Postgraduate Med. J., 52, 264-268.

BOURDET A., 1956a. Les protides des céréales. Ann. Techn. agric., 2, 253-266.

BOURDET A., 1956b. Les protides des céréales. Ann. Techn. agric., 2, 267-273.

CHARBONNIER L., 1970. Isolement de l'w-gliadine. C. R. Acad. Sci. Poris, 271, 2042-2045.

CHARBONNIER L., 1974. Isolation and characterization of $\omega$-gliadin fractions. Biochim. biophys. Acta, 359, 142-151.

CHARBONNIER L., MOSSÉ J., 1980. Large scale isolation of gliadin fractions. J. Sci. Fd Agric., 31 , 54-61.

CHEN C. H., BUSHUK W., 1970. Nature of proteins in Triticale and its parental species. Can. J. Plant Sci., 50, 9-14.

DICKE W. K., 1950. Cœliakie. Thèse Univ. Utrecht.

DISSANAYAKE A. S., TRUELOVE S. C., OFFORD R. E., WHITEHEAD R., 1973. Nature of toxic components of wheat gluten in cœliac disease. Lancet, ii 709-710.

DISSANAYAKE A. S., TRUELOVE S. C., WHITEHEAD R., 1974. Lack of harmful effect of oats on small intestinal mucosa in coliac disease. Br. Med. J., 4, 189-191.

EWART J. A. D., 1967. Amino acid analyses of glutenins and gliadins. J. Sci. Fd Agric., 18, 111-116.

JOS J., LENOIR G., DE RITIS G., REY J., 1975. In vitro pathogenetic studies of cœliac disease. Scand. J. Gastroent., 10, 121-128.

JOS J., REY J., 1975. Apport de la culture organotypique à l'éfude pathogénique de la maladie cceliaque. Arch. fr. Mal. appl. Dig., 64, 461-464.

JOS J., CHARBONNIER L., MOUGENOT J. F., MOSSÉ J., REY J., 1978. In McNicholl B., McCarthy C. F., Fottrell P. F. Perspectives in cœliac disease, 75-90. MTP Press Ltd, Lancaster, England.

KASARDA D. D., 1978. The relationships of Wheat proteins to cœeliac disease. Cereal Foods World, 23, $240-244$. 
KIM S. I., 1978. Etude sur les protéines du caryotype de l'Avoine. Th. Doct. Etat, Univ. Paris VI.

KIM S. 1., CHARBONNIER L., MOSSÉ J., 1978. Heterogeneity of avenin, the oat prolamin. Fractionation, molecular weight and aminoacid composition. Biochim. biophys. Acta, 537, 22-30.

LAFORGUE-TERCÉ T., 1979. Isolement et caractérisation des $\beta$-gliadines de Blé. Th. Doct. $3^{e}$ cycle, Univ. Paris VI.

LANDRY J., SALLANTIN M., BAUDET J., MOSSÉ J., 1965. Extraction des protéines des graines. Ann. Physiol. vég., 7, 283-293.

LAURIÈRE M., CHARBONNIER L., MOSSÉ J., 1976. Nature et fractionnement des protéines de l'Orge extraites par l'éthanol, l'isopropanol et le n-propanol à des titres différents. Biochimie, 58, 1235-1245.

McCRAE W. M., 1970. In BOOTH C. C., DOWLING R. H. Cœliac disease, 55-61. Churchill Livingstone, Edinburgh.

MOSSÉ J., 1966. Alcohol-soluble proteins of cereal grains. Fed. Proc., 25, 1663-1669.

MYLOTTE M., EGAN MITCHELL B., FOTTRELL P. F., MCCARTHY C. F. and MCNICHOLL B., 1973. Incidence of cœliac disease in the West of Ireland. Br. Med. J., 4, 189-191.

PAGÉ M., GODIN C., 1969. On the determination of the molecular weight of protein subunits on Sephadex G 200 in the presence of detergent. Glutamate deshydrogenase. Can. J. Biochem., 47, 401-403.

PRESTON K. R., WOODBURY W., 1975. Aminoacid composition and subunit structure of rye gliadin proteins fractionated by gel filtration. Cereal Chem., 52, 719-726.

SHEWRY P. R., FIELD J. M., KIRKMAN M. A., FAULKS A. J., MIFLIN B. J., 1979. The extraction, solubility and characterization of two groups of barley storage polypeptides. J. exp. Bot. (in press).

SMYTH D. G., 1967. In Methods in Enzymology, XI, 216-231. Hirs CHW ed., Acad. Press N. Y. and London.

TOWNLEY R. R. W., BHATHAL P. S., CORNELL H. J., MITCHELL J. D., 1973. Toxicity of wheat gliadin fractions in cœliac disease. Lancet, 1, 1362-1364.

VAN DE KAMER J. A., WEIJERS H. A., DICKE W. K., 1953. An investigation into the injurious constituents of Wheat in connection with their action on patients with cœliac disease. Acta paediatr., 42, 223-231.

VAN DE KAMER J. A., WEIJERS H. A., 1955. Some experiments on the cause of the harmful effect of Wheat gliadin. Acta paediatr., 44, 465-469.

WOYCHICK J. K., BOUNDY J. A., DIMLER R. J., 1961. Starch gel electrophoresis of wheat gluten proteins with concentrafed urea. Arch. Biochem. Biophys., 94, 477-482. 